Rabaska

Revue d'ethnologie de l'Amérique française

\title{
Joseph-Thomas LeBlanc, correspondant acadien de Marius Barbeau
}

\section{Louis-Martin Savard}

Volume 13, 2015

Présence de Marius Barbeau : l'invention du terrain en Amérique

française. Autour d’un legs centenaire (1914-2014)

URI : https://id.erudit.org/iderudit/1033752ar

DOI : https://doi.org/10.7202/1033752ar

Aller au sommaire du numéro

Éditeur(s)

Société québécoise d'ethnologie

ISSN

1703-7433 (imprimé)

1916-7350 (numérique)

Découvrir la revue

Citer cet article

Savard, L.-M. (2015). Joseph-Thomas LeBlanc, correspondant acadien de

Marius Barbeau. Rabaska, 13, 89-97. https://doi.org/10.7202/1033752ar d'utilisation que vous pouvez consulter en ligne.

https://apropos.erudit.org/fr/usagers/politique-dutilisation/ 


\section{Joseph-Thomas LeBlanc, correspondant acadien de Marius Barbeau}

LOUIS-MARTIN SAVARD

Université de Moncton

Lorsque nous nous interrogeons à propos des personnalités qui ont marqué l'histoire de la recherche sur le folklore en Acadie, le premier nom qui nous vient généralement à l'esprit est celui du père Anselme Chiasson. Ce réflexe apparaît en tout point justifiable : bien audacieux serait d'ailleurs celui qui tâcherait de remettre en question les accomplissements du père capucin originaire de Chéticamp. Dans ce qui suit, il sera question d'un autre folkloriste acadien, celui-ci moins connu, mais, suivant d'autres paradigmes appréciatifs, tout aussi remarquable : Joseph-Thomas LeBlanc.

La fin des années 1930 et le début de la décennie suivante constituent une période faste pour l'histoire de l'ethnographie en Amérique française. Pour ne citer que quelques exemples liés sur un plan synchronique à notre propos, retenons que c'est en 1937 qu'est publié Le Romancero du Canada ${ }^{1}$ de Marius Barbeau ; et, un peu plus tard, que l'année 1944 marque la fondation, par Luc Lacourcière et Félix-Antoine Savard, des Archives de folklore de l'Université Laval. À la même époque, en Acadie, au moment où la cathédrale Notre-Dame de l'Assomption, monument officiel de la « Reconnaissance ", est érigée, un homme s'intéresse à la recherche sur la chanson de tradition orale. Malgré des ressources parfois modestes, Joseph-Thomas LeBlanc, journaliste de Moncton, souhaite collaborer à cette vaste entreprise patrimoniale et culturelle qui concerne tous les francophones d'Amérique².

1. Marius Barbeau, Romancero du Canada, Toronto, MacMillan, 1937, 254 p.

2. LeBlanc conçoit une méthode d'investigation inédite en convoquant ses lecteurs à lui envoyer des transcriptions manuscrites de chansons. À distance, il essaie d'aiguiller ces derniers par des directives claires et appropriées à la collecte ethnographique. Comme il le spécifie : « Les personnes qui nous enverraient des vieilles chansons voudront bien les écrire d'un seul côté du feuillet et ne pas essayer de les rédiger en français moderne. Il faut essayer de les écrire telles qu'elles se chantent ». (Joseph-Thomas Leblanc, « Nos vieilles chansons acadiennes : La Barbière de Paris », Moncton, La Voix d'Évangéline, 16 mars 1939, p. 11.) À partir des chansons reçues, sont rédigées de courtes études ou chroniques. 
Entre 1938 et 1941, Joseph-Thomas LeBlanc fait publier 87 chroniques $^{3}$ intitulées « Nos vieilles chansons acadiennes » dans les pages du périodique néo-brunswickois La Voix d'Évangéline. Dès les années 1920, LeBlanc s'était intéressé au patrimoine immatériel acadien et à la fin de la décennie 1930, il souhaite doter l'Acadie de son premier ouvrage critique portant sur la chanson de tradition orale, un « romancero acadien ", en quelque sorte. Malheureusement, LeBlanc est décédé prématurément en 1943 avant de pouvoir rendre à terme tous ses projets et de réaliser ses ambitions.

Nous possédons un nombre limité d'informations sur sa vie personnelle. Toutefois, l'examen des relations épistolaires qu'il a entretenues avec les divers acteurs du milieu ethnographique de son époque, notamment avec Marius Barbeau, nous permet de mieux comprendre ses visées professionnelles et par le fait même de mieux mesurer la véritable valeur de ses réalisations.

L'œuvre de LeBlanc, même inachevée, demeure colossale. Il a collecté 1875 versions de chansons de tradition orale. Nous pourrions ajouter à cet inventaire la présence de plus de 3000 fiches sur le parler acadien qu'il a rédigées tout au long de sa carrière.

\section{Voyage posthume à Ottawa}

Le 20 décembre 1946, LeBlanc est décédé depuis quelques années. Marius Barbeau écrit au père René Beaudry du Collège Saint-Joseph à Memramcook. Dans sa lettre, nous pouvons lire :

Puis-je vous demander de nous prêter la collection folklorique de Jean-Thomas [sic] LeBlanc, comme vous l'avez offert lors de mon passage à votre université. Nous voudrions savoir au juste ce que contient cette collection, en sus de ce que LeBlanc lui-même m'a communiqué au cours du temps. Luc Lacourcière, François Brassard et moi-même, nous nous intéressons vivement à faire valoir l'œuvre inachevée de LeBlanc, et aussi à en faire profiter nos grandes collections ${ }^{4}$.

C'est ainsi que la majeure partie ${ }^{5}$ des collectes de chansons de LeBlanc seront envoyées à Ottawa pour être retranscrites. Elles retourneront en Acadie par la suite. Dans les décennies subséquentes, de nombreux chercheurs mettront à profit cette collection incomparable.

3. 88 chroniques, si nous considérons un premier texte ne portant pas l'intitulé « Nos vieilles chansons acadiennes $»$.

4. CÉAAC, Fonds J.-Thomas LeBlanc, M. Barbeau au père R. Baudry, 20 décembre 1946.

5. En 1946, ce sont 1340 versions de chansons qui sont transmises au Musée National à Ottawa, aujourd'hui Musée canadien de l'histoire. 535 autres versions n'ont jamais été envoyées dans la capitale et demeurent exclusives au Centre d'études acadiennes Anselme-Chiasson (CÉACC) de l'Université de Moncton. 
Afin de bien saisir la teneur de cette requête, un retour en arrière s'impose. Dans ce qui suit, nous verrons comment Barbeau a exercé une influence indéniable sur son homologue acadien, mais aussi nous soulignerons comment le travail de LeBlanc, par sa qualité et par son originalité, est parvenu à déteindre sur ses contemporains.

\section{Un important soutien}

À la fin de l'été 1938, LeBlanc envoie une première lettre à Barbeau et lui expose ses projets. La réponse, fort positive, laisse présager une collaboration féconde. Lettre après lettre, celui qui exerce l'autorité en matière de recherches ethnographiques à Ottawa encourage et approuve ouvertement le travail que poursuit le rédacteur adjoint de La Voix d'Évangéline. Se reporter aux missives que se sont échangées Barbeau et LeBlanc, c'est ainsi comprendre l'importance de l'indubitable soutien symbolique qu'a rapidement reçu le journaliste-folkloriste.

Le 25 novembre 1938, Barbeau lui écrit : « [V]ous allez devenir un de nos meilleurs collectionneurs de chansons, avec Massicotte et Lambert. Je vois que vous avez à votre disposition un domaine d'une grande richesse et encore inexploité. ${ }^{6} \gg$ Quelques mois plus tard, le 27 mars 1939, il poursuit : «Vraiment je suis étonné et émerveillé de la richesse de votre cueillette qui, je le vois, va longtemps se continuer. ${ }^{7} »$

En 1938, lorsque s'amorce l'échange épistolaire entre les deux hommes, Barbeau vient tout juste de faire publier son Romancero du Canada. Il profite de l'occasion pour en acheminer un exemplaire à son correspondant néo-brunswickois. Avec clémence et bienveillance, il autorise même ce dernier à citer son ouvrage à l'intérieur de ses chroniques :

J'y ai remarqué, à un certain endroit, que vous regrettiez de ne pas pouvoir reproduire des passages du ROMANCERO à cause de la petite clause des droits d'auteur au commencement $[s i c]$. Je puis vous dire, une fois pour toutes que vous pouvez en reproduire tout ce que vous voudrez avec ma permission et la permission implicite des éditeurs. ${ }^{8}$

En plus de son propre livre, Barbeau, à la fois mentor et protecteur, expédie à LeBlanc d'autres ouvrages de référence. « Je vais vous envoyer le romancéro de Doncieux ${ }^{9}$ que notre bibliothèque est prête à vous prêter, et je vous enverrai aussi d'autres recueils de notre bibliothèque. Ces livres vous seront envoyés comme prêt exceptionnel et à titre de collaborateur. ${ }^{10}$ »

6. CÉAAC, Fonds J.-Thomas LeBlanc, M. Barbeau à J.-Thomas LeBlanc, 25 novembre 1938.

7. CÉAAC, Fonds J.-Thomas LeBlanc, M. Barbeau à J.-Thomas LeBlanc, 27 mars 1939.

8. CÉAAC, Fonds J.-Thomas LeBlanc, M. Barbeau à J.-Thomas LeBlanc, 30 janvier 1939.

9. George Doncieux, Le Romancéro populaire de la France, Paris, Émile Bouillon, 1904, $522 \mathrm{p}$.

10. CÉAAC, op. cit., 30 janvier 1939. 
Ajoutons que c'est par l'entremise de Barbeau que LeBlanc prend connaissance de la collection des abbés Arsenault et Gallant recueillie à Île-duPrince-Édouard dans les années $1920^{11}$. Cette dernière, constituée d'environ 130 chansons, représente la première collecte de chansons de tradition orale en Acadie.

Maintes informations issues de ce corpus de référence, comme autant de points de repère, se montrent grandement utiles pour l'apprenti folkloriste. Dans les chroniques subséquentes à la réception du colis, elles sont abondamment citées et prises en exemple. Avant cela, le seul outil dont semble disposer LeBlanc, du moins le seul qu'il cite, est le recueil d'Ernest Gagnon $^{12}$.

Enfin, Barbeau affirme ouvertement son intention de couvrir LeBlanc de son égide afin que celui-ci bénéficie d'une bourse lui permettant d'avancer ses recherches et ultimement de faire publier son propre recueil de chansons.

Si vos circonstances (position et travail) s'y prêtaient, il serait possible de faire une demande pour vous (avant l'hiver prochain) d'une bourse de la Société royale, fondation Carnegie, comme celle qu'a reçue Lacourcière ; ou encore une bourse plus considérable aux E.-U. [...] pour la préparation d'un livre de chansons - les vôtres. Mais il faudrait, au cas où vous les obteniez, vous absenter, à l'étranger. ${ }^{13}$

Sans contredit, l'excellent rapport qu'a entretenu LeBlanc avec Marius Barbeau témoigne et valide la pertinence des chroniques " Nos vieilles chansons acadiennes ».

\section{LeBlanc était-il l'émule de Marius Barbeau?}

Pour articuler ses analyses, LeBlanc s'inspire largement de la démarche empruntée par son aîné. Parfois même, avec l'accord de ce dernier, il inclut dans ses textes des passages du Romancero du Canada ${ }^{14}$. Du reste, la méthode qu'il adopte varie semaine après semaine et nous pouvons difficilement $\mathrm{y}$ discerner une progression clairement jalonnée de changements significatifs qui nous permettrait de la découper en périodes bien distinctes. Néanmoins, constatons que plus la publication avance, plus le Monctonien gagne en assurance et développe une technique qui lui est propre. En quelque sorte, l'écriture des chroniques lui permet, dirions-nous, de se " faire la main ", d'affiner son discours critique et de s'adonner à des expérimentations sur le

11. Musée canadien de l'histoire, coll. abbé Pierre-Paul Arsenault.

12. Ernest Gagnon, Chansons populaires du Canada (deuxième édition), Québec, Robert Morgan, 1880.

13. CÉAAC, Fonds J.-Thomas LeBlanc, M. Barbeau à J.-Thomas LeBlanc, 13 mars 1940.

14. Marius Barbeau, op. cit. 
plan méthodologique. Il importe de ne pas perdre de vue qu'à cette époque LeBlanc vise plus grand que la simple publication de textes journalistiques; son souhait le plus cher est de doter l'Acadie de son premier recueil critique de chansons de tradition orale ${ }^{15}$. Cela dit, dès les premières chroniques, au moment où il entre en contact avec Barbeau, son travail est rapidement reconnu, voire formellement encouragé et louangé. Au fil du temps, leur relation se développe. Dans une juste mesure, nous pourrions affirmer que ces façons de faire, ces chemins empruntés par LeBlanc vont jusqu'à déteindre sur l'auteur du Romancero du Canada. Rapidement, celui-ci n'hésite pas à gratifier son homologue acadien de propos élogieux : "Vous dites que mes "notes et contextes sont fort intéressants" oui, mais je dois avouer en toute franchise qu'ils s'inspirent de vos notes et vos contextes à vous. Sous ce rapport, c'est vous qui êtes, en quelque sorte, mon maître ${ }^{16}$. » Quelques années après le décès de LeBlanc, c'est Barbeau qui, paradoxalement, inclura des passages de la chronique « Nos vieilles chansons acadiennes» dans ses écrits ${ }^{17}$.

Nous l'avons mentionné, les chroniques ne se structurent pas toutes suivant le même canevas. LeBlanc traite parfois de la dimension thématique des chansons, passages où il apporte des précisions sur la signification des paroles. Lorsque la nature du sujet s'y prête, à ces gloses succède souvent un commentaire servant à contextualiser ce que raconte le texte chansonnier. À d'autres moments, LeBlanc met plutôt l'accent sur des aspects formels du texte ; il souligne des traits concernant les modalités de versification et d'assonance des pièces, ce qui rappelle d'ailleurs la méthode de Barbeau. LeBlanc s'intéresse également à la langue. Ainsi, il ne manque pas d'établir des parallèles à teneur philologique entre les mots présents dans les chansons qu'il recueille et certains archaïsmes issus des vieux parlers régionaux de France. C'est d'ailleurs avec un enthousiasme probant qu'il décrit la valeur et les particularités plus locales du lexique employé par ses informateurs, cela tout en s'efforçant de souligner les réseaux de filiation qui lient les chansons qu'il collecte à celles qui ont été retrouvées en France.

$[C]$ ertaines vieilles chansons de France semblent être inconnues en Acadie mais par contre, et c'est ce qui arrive le plus souvent, des chants populaires qui outremer, ont été complètement mis en oubli ou n'existent maintenant que sous

15. Pour plus de détails, voir les articles suivants : Charlotte Cormier et Donald Deschênes, « Joseph-Thomas LeBlanc et le romancero inachevé », Canadian Folklore canadien, vol. 13, n 2, 1991, p. 55-70 ; Louis-Martin Savard, « Joseph-Thomas LeBlanc : la chanson de tradition orale au pied de la lettre », Rabaska, revue d'ethnologie de l'Amérique française, vol. 10, 2012, p. 47-68.

16. CÉAAC, Fonds J.-Thomas LeBlanc, M. Barbeau à J.-Thomas LeBlanc, 15 mai 1939.

17. Marius Barbeau, « Rossignol du vert bocage », L'Action Catholique, Québec, vol. x, n 36, 8 sept. 1946 , p. 6. 
forme de fragments, se retrouvent aujourd'hui, aux Provinces Maritimes, dans leur forme intégrale. ${ }^{18}$

LeBlanc est conscient de la richesse de l'Acadie des Maritimes en tant que terrain d'investigation ethnographique et c'est non sans un certain orgueil qu'il en vante les mérites.

\section{Les variantes et les versions}

Surtout dans les premières chroniques, à l'instar de bien des contemporains et prédécesseurs, LeBlanc tente d'établir des versions esthétiques de chansons. Il est vrai que, dans les années où LeBlanc conçoit ses chroniques, les folkloristes favorisent généralement la publication de versions esthétiques, une méthode qui consiste à forger, à partir de plusieurs variantes d'une même chanson, un texte qu'on nommera « critique » ou « esthétique ». À ce sujet, Barbeau, et plus tard Lacourcière compareront souvent leur démarche à celle d'un archéologue essayant de reconstituer un vase brisé à partir de fragments disséminés. Cette analogie illustre bien une école de pensée issue des travaux du philologue français Gaston Paris et adaptée au domaine de la chanson de tradition orale par son disciple George Doncieux. Longtemps favorisées pour sa nature propice aux démonstrations logiques, la méthode de la philologie appliquée à l'étude de la chanson est aujourd'hui contestée. À son époque, LeBlanc ne fait pas exception et adhère en grande partie aux théories sur lesquelles s'appuient les travaux de bon nombre de ses contemporains. Dans une lettre qu'il envoie à Barbeau le 2 septembre 1938 il écrit : « il faudra des années de recherches et aussi il me faudra comparer les différents textes recueillis avant de pouvoir reconstituer convenablement les textes de chansons de "sur l'en-premier"19 ». Sans équivoque, LeBlanc semble partager les idées et les méthodes que véhicule Barbeau. Toutefois, ce qui étonne, c'est comment il s'en détourne.

À cette méthode de la version esthétique, LeBlanc préfère la présentation des chansons dans la diversité de leurs variantes. C'est notamment pour cette raison, pouvons-nous supposer, que « Nos vieilles chansons acadiennes » retiennent rapidement l'attention, non seulement des lecteurs du journal, mais aussi des spécialistes. Très souvent les textes qu'il propose ne sont que très peu altérés, ce qui augmente singulièrement leur valeur documentaire.

Barbeau le fait remarquer, la présence des variantes ajoutée aux contraintes de l'édition peut, dans certains cas, dicter la méthode qu'emploiera le chercheur : « [v]oilà l'écueil qui menace tout folkloriste, lorsqu'il veut

18. Joseph-Thomas LeBlanc, « La Jeune Bergère », La Voix d'Évangéline, 11 avril 1940, p. 11.

19. CÉAAC, Fonds J.-Thomas LeBlanc, J.-Thomas LeBlanc à M. Barbeau, 2 septembre 1938. 
présenter une chanson ancienne dont les versions sont nombreuses et accidentées : ou bien il doit les citer au complet une à une, ce qui est onéreux et oiseux, ou bien il doit en rédiger un texte moyen ou critique $!^{20} »$ D'un point de vue strictement éditorial, la version esthétique est donc justifiable.

Il n'en demeure pas moins que, dès les années 1920, cette méthodologie ainsi que les a priori qui l'accompagnent sont critiqués. Arnord Van Gennep, inspiré par Julien Tiersot, écrit alors :

$[\ldots]$ certaines tentatives de reconstitution des formes primitives d'une chanson, par exemple de la Pernette, par la comparaison des diverses variantes, tentative dont la plus intéressante est celle de Doncieux dans son Romancero populaire (Champion). Il a essayé d'éliminer les éléments adventices ou secondaires des variantes au profit d'un texte littéraire et mélodique qu'il a regardé comme celui qui avait été inventé, le premier. Mais il n'a pu faire cette élimination qu'à l'aide d'arguments purement personnels et subjectifs, et en fin de compte, malgré le soin méticuleux de sa recherche, il n'a convaincu personne. ${ }^{21}$

Évidemment, du fait qu'elle envisage la chanson comme une œuvre issue d'un auteur unique, la version esthétique ne s'accorde pas à la diversité inhérente à toute tradition orale. De même, sa dimension factice trahit ce que nous pourrions appeler « la vérité du terrain ». Remarquons au passage que le grand folkloriste français Patrice Coirault ne lui a jamais été favorable.

Nous l'avons mentionné, LeBlanc s'adonne néanmoins à cette méthode. Toutefois, et peut-être malgré lui, il la met très souvent de côté afin de favoriser la publication de plusieurs variantes de la même chanson. En réalité, dès ses premières chroniques, il manifeste une évidente propension pour la diffusion des variantes. Qu'est-ce qui le motive à procéder ainsi ? Le fait que le nom des informateurs soit inscrit dans le journal ? Il est vrai que LeBlanc identifie toujours les noms et prénoms de ceux et celles qui lui transmettent des chansons. Modifier les textes de ces précieux informateurs pourrait être interprété comme un manque de respect, d'autant plus que LeBlanc ne rate jamais une occasion de remercier chaudement ceux qui participent à l'édification du répertoire des vieilles chansons acadiennes. Il leur exprime sa gratitude en public, dans le journal, mais également en privé. L'extrait suivant tiré d'une lettre qu'il adresse à Barbeau met en lumière toute la reconnaissance que l'auteur des chroniques éprouve à l'égard de ses collaborateurs :

Je vous envoie ce texte surtout afin que vous le consigniez aux archives à Ottawa et il me vient, en même temps, une idée : pourquoi, le département des Archives à Ottawa ne songerait-il pas à rémunérer $\mathrm{M}^{\mathrm{me}}$ Noël, d'avoir conservé

20. Marius Barbeau, « Trois beaux canards », Les Archives de folklore, Montréal, Fides, vol. 2, 1947, p. 100.

21. Arnold Van Gennep, Le Folklore, Paris, Stock, 1924, p. 38. 
une aussi précieuse chanson. Certes, cela vaut certainement quelque chose et moi-même, si j'étais riche, je n'hésiterais pas à récompenser $\mathrm{M}^{\text {me }}$ Noël, mais malheureusement je ne puis le faire. ( $\mathrm{M}^{\mathrm{me}}$ Noël ne demande aucune rémunération, mais tout de même il faudrait lui savoir gré de sa collaboration à notre folklore). Je consider [sic] qu'il s'agit ici d'une trouvaille peu ordinaire. Moimême, je ne veux pas de récompense, mais je serais heureux de voir $\mathrm{M}^{\mathrm{me}}$ Noël obtenir quelque chose en retour de sa contribution. Je soulignerai en passant que notre gouvernement fédéral fait souvent de fortes dépenses pour des choses qui ont bien moins d'importance que nos documents folkloriques. Et cela, le gouvernement fédéral le fait sans hésitation ${ }^{22}$.

Cela dit, LeBlanc, lorsqu'il publie une variante de chanson qu'un informateur lui a envoyée et qu'il indique explicitement par une formule du type « la version suivante nous provient de... », ne s'adonne généralement qu'à de très légères modifications. Il rectifie l'orthographe et la grammaire. Il change parfois un mot inconnu ou une expression incohérente, rien de plus. Dans la majorité des cas, il demeure fidèle au texte qu'on lui a fait parvenir. Pour plusieurs chansons, il reçoit des versions partiellement identiques. Dans ces situations, il sélectionne celle qui lui apparaît la plus commune, à laquelle il donne, ou non, une facture plus « esthétique ». Il s'efforce cependant de toujours présenter les segments qui diffèrent de la version « principale » et d'en identifier la provenance. Il pratique plus ouvertement la version esthétique lorsqu'à partir d'une version partielle d'une chanson venant la plupart du temps de son répertoire personnel, il tente de reconstituer un texte plus complet. Cette méthode n'est toutefois vraiment pas généralisée. Ce que nous devons retenir, c'est avant tout le nombre substantiel de variantes et de versions de chansons qui ont été diffusées par le biais des chroniques. Par exemple, pour Jean Renaud [II.A.I], chanson tragique par excellence, six versions complètes ont été publiées. De plus, spécifions qu'à maintes reprises les chansons que recueille et diffuse LeBlanc diffèrent de celles déjà collectées à l'époque. Dans une lettre qu'il envoie à Barbeau en 1940, Lacourcière écrit : «Un autre point qui m'embarrasse un peu, ce sont les nouvelles versions de LeBlanc qui viennent un peu changer mes listes de variantes, mais c'est un point secondaire. Dans certains cas je pense qu'il faudra une rédaction pour les versions acadiennes ${ }^{23}$. »

\section{Le journal, pierre angulaire des recherches}

Même si, par la nature de leur mode de diffusion, les textes s'adressent d'entrée de jeu au grand public, les chroniques sont tenues en estime par les spécialistes. Par exemple, le 22 avril 1940 (à ce moment, environ

22. CÉAAC, Fonds J.-Thomas LeBlanc, J.-Thomas LeBlanc à M. Barbeau, 20 juin 1939.

23. Afeul, Fonds P178/C5, L. Lacourcière à M. Barbeau, 28 février 1940. 
65 chroniques ont été publiées), François Brassard, qui quelques années plus tard se joindra à l'équipe des Archives de folklore de l'Université Laval, écrit à LeBlanc : " J'ai suivi avec grand intérêt l'enquête que vous avez menée dans votre journal sur la chanson acadienne. J'ai tenu à en conserver la série au complet ${ }^{24} »$. Nous venons d'emprunter les mots de François Brassard, mais nous pourrions tout autant citer Lacourcière ou Barbeau. Nous attirerons par ailleurs l'attention sur le mot « enquête » utilisé par Brassard, car LeBlanc, utilisant les simples moyens dont il dispose, s'adonne à de véritables enquêtes.

En fait, si la correspondance épistolière permet au chercheur d'aujourd'hui de mieux comprendre et de pouvoir disséquer le travail du folkloriste, à l'époque où ce dernier prépare ses chroniques, elle aura une importance capitale dans la manière avec laquelle il collectera ses chansons. Le terrain d'enquête exploré par LeBlanc le sera majoritairement par le biais de lettres échangées entre lui et ses informateurs, et c'est par l'intermédiaire des pages du journal qu'il établira le contact avec ces derniers.

Dans les années durant lesquelles il rédige ses chroniques, LeBlanc transmet avec assiduité les résultats de ses investigations. Marius Barbeau et bien d'autres en profiteront par la suite. En 1946, à la demande de Barbeau, le fonds LeBlanc est prêté à Ottawa afin d'y être retranscrit, ce qui permettra à de nombreux ethnologues de reconnaître sa richesse et de le mettre à profit dans leurs recherches. Il serait difficile d'en dire autant des chroniques.

\section{Conclusion}

Parues entre le 28 juillet et le 27 mars 1941, les chroniques « Nos vieilles chansons acadiennes » auront laissé leur marque. Toutefois, elles demeurent aujourd'hui méconnues, même de la part de nombreux spécialistes et ethnologues. Pourtant, celles-ci, dans le contexte de leur époque, font preuve d'une originalité incontestable. LeBlanc, à partir d'une technique de collectage inédite, $\mathrm{y}$ a présenté et étudié la chanson de tradition orale suivant des modalités uniques en leur genre. Aussi, il n'est pas insensé d'avancer qu'il a inspiré des ethnologues reconnus comme Barbeau et Lacourcière, notamment par sa façon de faire avec les variantes. Évidemment - et cela reste à démontrer -, celui qui prend le temps de comparer les chroniques à des études classiques telles que «Les Écoliers de Pontoise $»^{25}$ de Lacourcière et les $« 92$ versions de Trois beaux canards $»^{26}$ de Barbeau peut difficilement s'empêcher d'en percevoir les traits communs.

24. CÉAAC, Fonds J.-Thomas LeBlanc, François Brassard à Joseph-LeBlanc, 22 avril 1940.

25. Luc Lacourcière, «Les Écoliers de Pontoise, étude critique d'une chanson populaire », Les Archives de folklore, Montréal, Fides, vol. 1, 1946, p. 176-199.

26. Marius Barbeau, "Trois beaux canards (92 versions canadiennes) », Les Archives de folklore, Montréal, Fides, vol. 2, 1947, p. 97-138. 